\title{
Guest Editorial: Multimedia in Social Mobile Computing (MSMC)
}

\author{
Fernando Ferri $^{1}$ - Patrizia Grifoni ${ }^{1}$ - Arianna D'Ulizia ${ }^{1}$. \\ Maria Chiara Caschera ${ }^{1}$
}

Published online: 22 February 2017

(C) Springer Science+Business Media New York 2017

\section{Introduction}

The special issue on Multimedia in Social Mobile Computing (MSMC) was organised aiming to stimulate cross-disciplinary debate on researches involving multimedia technologies and social mobile computing.

The amount of multimedia data accessible from mobile devices is increasing more and more over the years, and new challenges on data management, situation-awareness, personalization, privacy, and security are emerging. The variety of multimedia data enables new applications across different research domains comprising mobile media analysis, mobile social networks, mobile human-computer interaction and multimodal issues [2, 3]. The community of researchers is increasingly investigating issues related to the pervasive use of mobile devices (e.g. Smartphone, PDA, GPS navigation and laptops) and wireless technology; mobile computing is experiencing a phase of strong innovation and, multimedia data and content are spreading over the network of mobile technologies. All these different aspects are studied in the perspective of mobile technologies and the collaborative multimedia systems that use devices interconnected through an ad-hoc network.

In this direction, social networks and content-sharing web systems are increasingly emerging as platforms that facilitate the creation and sharing of multimedia content and supporting new types of interaction. The growth of multimedia social systems stimulates researches on social computing that investigate emerging issues and challenges in modelling, analyzing and effectively interacting with social media.

That is why the topic of social multimedia computing is attracting researchers in multimedia computing and social sciences, as well as entrepreneurs producing software and mobile devices and applications.

Fernando Ferri

fernando.ferri@irpps.cnr.it 
For this special issue twenty papers were submitted. Eight of them were accepted after a rigorous review process that consisted of several rounds of review. During this process, the papers were blindly reviewed by at least three anonymous reviewers.

The eight selected papers address various issues connected with personalization and situation awareness for mobile multimedia, security in mobile multimedia, mobile multimedia applications and services, location-based social networks and media, and multimedia user interaction for mobile devices.

\section{Summary of the accepted papers}

The contributions of the accepted papers can be summarized as follows.

The paper titled "A comparative study of user intention to recommend content on mobile social networks" [4] explores user intention to recommend multimedia content on mobile social networks utilizing user behavioural responses on social network services. Authors analyze the intent to recommend services in the context of mobile social networks by integrating the theory of planned behaviour and the technology acceptance model. They state that social networks used by mobile devices increase the influence of heavy users in terms of subjective norm, trust, perceived ease of use, and perceived usefulness. Their study demonstrates that: 1) trust affects perceived ease of use and perceived usefulness, 2) perceived ease of use affects perceived usefulness, and 3) subjective norm drives trust for both heavy and light users. Their results for heavy users provide advanced guidelines for mobile social networks providers to understand the paths of perception on user intention to recommend with regard to light users.

The paper titled "An efficient reversible data hiding method for AMBTC compressed images" [5] proposes a type II reversible data hiding method for the absolute moment block truncation coding compressed code. Authors propose: 1) median edge detection and alternative prediction of the quantization values to improve the efficacy of prediction and 2) a centralization error division technique to divide prediction errors making it more efficient. Indeed, fewer bits are required to encode the prediction errors within the proposed method. The experiment results show that the proposed MAC method offers a comparable embedding capacity while providing a lower bit-rate and higher efficiency than methods described in other related works.

The paper titled "Improving quality of multimedia services through network performance isolation in a mobile device" [7] proposed MuSNet (a scheme for improving quality of service and quality of experience of multimedia services through network performance isolation in a mobile device), which provides network performance isolation for the multimedia service by mobile device-based control. The proposed schema allows managing wireless traffic in terms of processes and guaranteeing the network performance of the multimedia service when multiple Internet services run concurrently. In addition, MuSNet flexibly adjusts weight factors of control parameters and guarantees higher service quality for the multimedia service and maintains the overall performance of a device. MuSNet was implemented on a smart phone by modifying the kernel, and the performance of MuSNet was evaluated with in-depth analysis of various experiments using mobile devices.

The paper titled "A methodological approach for designing and developing web-based inventories of mobile Assistive Technology applications" [6] provides an analysis of the disabled users' needs along with the required mobile software adaptations in order to fulfill them. The authors introduce a methodological approach for the design and development of web-based inventories, which makes the search and selection of Assistive Technology apps 
simpler and efficient. This methodology was tested by a team of experts in an Assistive Technology lab. The paper provides a consistent and well-documented presentation of the information for each application. In addition, authors present the mATHENA repository of free Assistive Technology applications for mobile devices (smart phones and tablets), which has been created by following the suggested methodology.

The paper titled "SNS User Classification and its Application to Obscure POI Discovery" by Zhuang et al. [10] investigates the problem of mining the information asymmetry regarding location among various user groups of social networking services. To solve this problem, it proposes three models for automatically estimating the user's "location authority" (i.e. the user's familiarity with a particular location): (i) a social-network driven model, where a graph-based method is used to determine whether a user is living in the target city, (ii) a time-driven model, which estimates the user's location authority based on the frequency of visits, and (iii) a locationdriven model, which simulates collective mobility behaviour. By using these user-profiling models, the paper proposes a new application for geo-social recommendations that allows discovering points of interest (POIs) that are not well known, even though they are worth visiting.

In the paper titled "Hierarchical Threshold Secret Sharing Scheme for Color Images" by Fathimal and Rani [8], a novel disjunctive hierarchical secret sharing scheme is proposed, where secret information is divided into several pieces, with or without modifications, and is retrieved by combining predefined sub collection of pieces. The proposed scheme is for a two level hierarchical structure and it uses simple arithmetic calculations with reduced computational complexity (from $\mathrm{O}(\mathrm{n} \log 2 \mathrm{n}$ ) to $\mathrm{O}(\mathrm{n})$ ).

The paper titled "Assisstive technology application for enhancing social and language skills of young children with autism" by Wojciechowski and Al-Musawi [9], investigates how the technology can assist autistic children in learning pronunciation and meanings of new words and proposes a novel assistive system that is built-up of a mobile application and uses Estimote Beacons to identify objects. The system allows an autistic child to learn objects' names or sounds by hearing the words pronounced by a parent. The sound is played on a smart device and a cartoon image can be displayed repetitively whenever the smart device encounters and recognize an object equipped with a beacon. The experimental validation demonstrates that autistic children made a visible progress in speed of learning new words by using the system when compared to an equivalent period of time without assistive application support.

The paper titled "A Social Interactive Whiteboard System Using Finger-Tracking for Mobile Devices" [1] proposes a social interactive whiteboard system using finger-tracking for mobile devices. The application is a drawing tool, allowing users to create a virtual whiteboard and draw different kinds of lines, erase lines, zoom etc. from mobile devices that are featured with built-in projectors. In addition, authors investigate the problematic of tracking a finger using the build-in camera and how images are properly projected as a whiteboard from a mobile device. They present a thorough analysis of the various techniques that can be used to track a finger and depict our proposal to build the mobile-based interactive whiteboard application. Finally, the experimental evaluation analyzes the performance, reliability and usability of the application taking into account valuable feedback for end users.

\section{Conclusion}

The special issue has collected high quality contributions addressing cross-disciplinary researches between multimedia technologies and social mobile computing discussing 
technological opportunities, challenges, solutions, and emerging theories and applications in mobile social multimedia.

Acknowledgements We would like to express our gratitude to all the authors who have submitted their work for publication in this special issue. We are also thankful to all the reviewers that offered their time and competences to provide valuable peer reviews. Finally, we want to thank the Editor in Chief Borko Furht and all the Editorial Staff of the Multimedia Tools and Applications (MTAP) journal for their kind support for the processing of the publication of this special issue.

\section{References}

1. Bested M, Weisberg AH, Durão FA (2016) A social interactive whiteboard system using finger-tracking for mobile devices. Multimed Tools Appl. doi:10.1007/s11042-016-3922-0

2. Caschera MC, Ferri F, Grifoni P (2007) Multimodal interaction systems: information and time features. International Journal of Web and Grid Services (IJWGS) 3(1):82-99. doi:10.1504/IJWGS.2007.012638

3. Caschera MC, D’Ulizia A, Ferri F, Grifoni P (2015) Multimodal systems: an excursus of the main research questions, on the move to meaningful internet systems: OTM 2015 Workshops, Volume 9416 of the series Lect Notes Comput Sci, pp. 546-558, 2015. doi:10.1007/978-3-319-26138-6_59

4. Chang SE, Shen WC, Yeh CH (2016) A comparative study of user intention to recommend content on mobile social networks Multimed Tools Appl. doi:10.1007/s11042-016-3966-1

5. Hong W, Ma Y-B, Wu H-C, Chen T-S (2016) An efficient reversible data hiding method for AMBTC compressed images. Multimed Tools Appl. doi:10.1007/s11042-016-4032-8

6. Kouroupetroglou G, Pino A, Riga P (2016) A methodological approach for designing and developing webbased inventories of mobile assistive technology applications. Multimed Tools Appl. doi:10.1007/s11042016-3822-3

7. Lee W, Kim H, Lee JY, Kim H (2016) Improving quality of multimedia services through network performance isolation in a mobile device. Multimed Tools Appl. doi:10.1007/s11042-016-3821-4

8. Mohamed Fathimal P, Arockia Jansi Rani P (2016) Hierarchical threshold secret sharing scheme for color images. Multimed Tools Appl. doi:10.1007/s11042-016-4074-y

9. Wojciechowski A, Al-Musawi R (2016) Assisstive technology application for enhancing social and language skills of young children with autism. Multimed Tools Appl. doi:10.1007/s11042-016-3995-9

10. Zhuang C, Ma Q, Yoshikawa M (2016) SNS user classification and its application to obscure POI discovery. Multimed Tools Appl. doi:10.1007/s11042-016-4034-6 